\title{
Spontaneous cholecystectomy
}

\author{
J. C. MCGregor \\ B.Sc., M.B., Ch.B., F.R.C.S.
}

\author{
D. J. SAMMON \\ M.B., Ch.B., D.Obst., R.C.O.G., F.R.C.S.
}

Stobhill General Hospital, Glasgow

\begin{abstract}
Summary
A young female, who presented with acute appendicitis and peritonitis, is described. Following laparotomy she developed a series of intraperitoneal abscesses. After drainage of a subphrenic abscess a necrosed gallbladder was discharged several days later through the drainage wound in the abdominal wall.
\end{abstract}

\section{Case report}

Miss K.S., aged 12 years, was admitted on 8 January 1973 with a $48 \mathrm{hr}$ history of generalized abdominal pain of sudden onset associated with anorexia and constipation. Clinically she appeared to have peritonitis and a straight X-ray of her abdomen revealed multiple small bowel fluid levels. Her temperature was $39.4^{\circ} \mathrm{C}$ and her white blood count was 24,000 cells $/ \mathrm{mm}^{3}$. She was not observed to be jaundiced and gave no history suggestive of previous gallbladder disease or peptic ulceration. Laparotomy on the day of admission revealed a friable gangrenous appendix in close opposition to the ileal mesentery. The adjacent ileum was inflamed and distended and turbid peritoneal fluid was present. The appendix was carefully removed and the stump was oversewn. The abdominal wound was closed with drainage of the peritoneal cavity. Postoperatively she was treated with intramuscular ceporin but after 1 week she developed profuse diarrhoea and a temperature of $39^{\circ} \mathrm{C}$. A few days later a pelvic abscess discharged rectally and the laparotomy wound was noted to be infected. Escherichia coli was cultured from both sites and on the basis of sensitivity results the patient was commenced on gentamicin. Her temperature continued to be raised, however, and on 26 January 1973 further operative intervention was required to drain a right anterior subphrenic abscess and also a collection of pus in the left iliac fossa. She continued thereafter to make slow progress and required further drainage of the subphrenic abscess on 19 February 1973. On 27 February 1973 a piece of necrotic tissue appeared and discharged itself through this last wound. Pathology subsequently revealed this to be a necrotic gallbladder (Fig. 1). With continuing intensive therapy Miss K.S. gradually improved without re- quiring further surgical intervention. She was allowed home on 2 April 1973 and remains well to date.

\section{Discussion}

Spontaneous necrosis of the gallbladder is an uncommon condition. Gangrene associated with rupture following acute cholecystitis is not infrequent (Maingot, 1957). Infarction of a gallbladder, previously known to be macroscopically normal, is virtually unrecorded. Mitchell (1965) described a

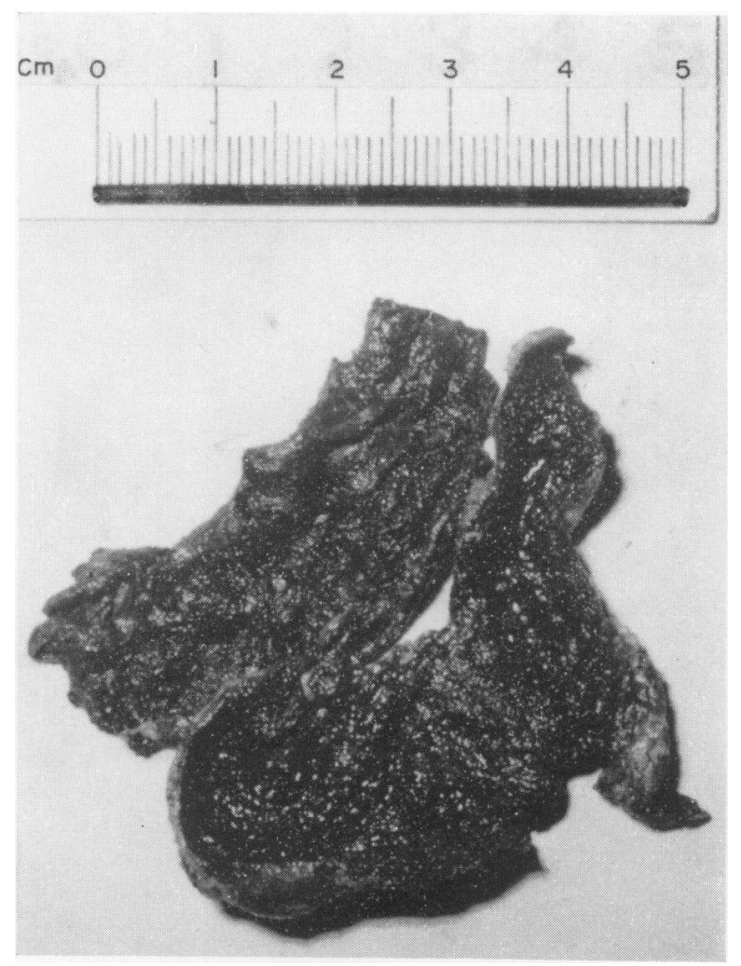

Fig. 1. Macroscopic appearance of the necrosed extruded gallbladder. The viscus has been opened out with the mucosal surface facing upwards and the fundus towards the bottom of the photograph. 
male patient in whom this occurred following development of multiple intra-abdominal abscesses after abdomino-perineal resection for carcinoma of the rectum. The author was unable to explain how this could occur especially as the region of the gallbladder appeared normal at post mortem.

The case described in this paper is another example of gallbladder necrosis following laparotomy and subsequent development of intraperitoneal abscesses. The histological appearance of the extruded gallbladder suggested that infarction had occurred. There was no direct evidence that the gallbladder was completely normal beforehand but in view of the age of the patient and the absence of any preceding history this would seem likely. It would appear not unreasonable to suggest that the presence of the subphrenic abscess in the region of the gallbladder, by causing thrombosis of the cystic artery and other vessels supplying the gallbladder, might have been associated with the production of an organ infarct.

The subsequent appearance and spontaneous discharge of a gallbladder through a surgical wound has not previously been reported. This, together with the rarity of infarction, makes the reported case unique.

\section{Acknowledgments}

We would like to thank Mr W. Rankin for permission to publish his case and also the Photographic Department of Stobhill Hospital, Glasgow, for the production of the photograph.

\section{References}

MaIngot, R. (1957) The Management of Abdominal Operations, p. 827. H. K. Lewis: London.

MitcheLl, J.E. (1965) Spontaneous necrosis of the gallbladder. Postgraduate Medical Journal, 41, 107. 